\title{
Distribución altitudinal de la riqueza y composición de "ensamblajes" de aves en una zona montañosa al sur de Nayarit, México
}

Nidia Gisell Jaime-Escalante ${ }^{1}$, Elsa Margarita Figueroa-Esquivel ${ }^{2 *}$, José Fernando VillaseñorGómez $^{3}$, Edwin Alberto Jacobo-Sapien ${ }^{1} \&$ Fernando Puebla-Olivares ${ }^{2}$

1. Posgrado en Ciencias Biológico Agropecuarias, Universidad Autónoma de Nayarit. Km. 9 Carretera TepicCompostela, CP 63780. Nayarit, México; nidia_gisell@hotmail.com, ed_jacobo@yahoo.com

2. Museo de Zoología, Unidad Académica de Agricultura, Universidad Autónoma de Nayarit. Km. 9 Carretera TepicCompostela, CP 63780. Nayarit, México; buco_figues@yahoo.com.mx, fernandopuebla@hotmail.com

3. Facultad de Biología, Universidad Michoacana de San Nicolás de Hidalgo, Edificio "R", Ciudad Universitaria, Gral. Francisco J. Múgica S/N, CP 58030 Michoacán, México; jfvillasenorg@hotmail.com

* Correspondencia

\author{
Recibido 27-VII-2015. Corregido 06-V-2016. Aceptado 09-VI-2016.
}

\begin{abstract}
Altitudinal distribution, richness and composition of bird "assemblages" in a mountainous region in Southern Nayarit, Mexico. Elevation gradient studies have strengthened the evaluation of changes in richness and composition of bird assemblages. They also provide information on environmental variables that determine bird distribution, and the variables that define their population structure. Our aim was to describe their variation through an elevational cline in Southern Nayarit, Mexico. To analyze the behavior of richness across the gradient, we gathered information through point counts in nine elevational intervals ( $300 \mathrm{~m}$ from each other) from sea level to $2700 \mathrm{~m}$ of elevation. With a standardized sampling effort, we produced rarefaction curves and analyzed changes in species composition by hierarchical classification using the TWINSPAN technique. In order to identify variables associated with richness changes, we examined the effect of precipitation and habitat structure via regression trees. An analysis of nonmetric multidimensional scaling (NMDS) was implemented with the purpose to determine if the changes in composition correspond to changes in vegetation types. Species richness varied significantly across the gradient: high in the lower parts of the gradient, reached its peak in the middle, and decreased monotonically with elevation. Species responded to changes in the cline and were grouped in three elevational zones. Analyses suggest that changes in richness and species composition are influenced by vegetation, its structure and precipitation regime, as well as various aspects related to habitat features and disturbance. These aspects should be taken into account in order to design appropriate strategies for the conservation of the birds of Nayarit. Rev. Biol. Trop. 64 (4): 1537-1551. Epub 2016 December 01.
\end{abstract}

Key words: distribution patterns, altitudinal gradients, bird communities, species richness, endemic species.

Desde las primeras exploraciones, los naturalistas observaron que los seres vivos no están homogéneamente distribuidos, y que contrario a esto, la riqueza de especies se comporta siguiendo patrones de distribución (Gaston, 2000; Koleff et al., 2008). Uno de los primeros patrones observados fue el cambio de la riqueza en un gradiente altitudinal, aceptándose ampliamente que disminuía conforme se incrementaba la elevación (Lomolino, 2001; McCain \& Grytnes, 2010). Sin embargo, investigaciones más recientes sobre el tema, han revelado que la riqueza de especies puede comportarse de acuerdo con cuatro patrones: 1) la riqueza disminuye monotónicamente con el aumento de la elevación (“disminución gradual”); 2) la riqueza es máxima y constante en las partes bajas y posteriormente disminuye monotónicamente con la elevación ("mayor riqueza en bajas altitudes"); 3) la riqueza es alta en elevaciones bajas, alcanza su máximo a mitad del gradiente $\mathrm{y}$ entonces disminuye 
monotónicamente con la elevación ("mayor riqueza en bajas altitudes con incremento en elevaciones intermedias"); 4) la riqueza es máxima a mitad del gradiente y disminuye hacia las partes bajas y altas ("mayor riqueza en altitudes intermedias") (Rahbek, 1995; McCain, 2009).

La frecuencia con la que se presenta cada patrón varía de acuerdo al grupo taxonómico estudiado, a los métodos utilizados y a distintos factores y procesos involucrados, como escala del estudio, extensión de las áreas, clima, productividad, factores bióticos o la historia evolutiva (Cadena et al., 2012; Rahbek, 1995; Hart et al., 2011; Herzog, Kessler, \& Bach, 2005; McCain \& Grytnes, 2010; Wilson, Valqui, \& McCracken, 2010), por lo que es claro que existen diversas manifestaciones del comportamiento de la riqueza de especies a través de gradientes altitudinales (Herzog et al., 2005). El análisis realizado por McCain y Grytnes (2010) muestra que entre los vertebrados, los cuatro patrones descritos se encuentran presentes: el patrón de "mayor riqueza en altitudes intermedias" correspondió al $45 \%$ de los casos, el de "disminución gradual" al $26 \%$, el de "mayor riqueza en bajas altitudes" al $15 \%$ y el de "mayor riqueza en bajas altitudes con incremento en elevaciones intermedias" al $14 \%$. En el caso particular del grupo de aves, McCain (2009), encontró que de un total de 78 estudios sobre gradientes montañosos, los cuatro patrones se presentaron en frecuencias similares.

El entendimiento de la relación entre la riqueza de especies y la elevación a nivel local o regional ayudaría a explicar los procesos que causan el patrón observado (Kattan \& Franco, 2004). Actualmente, el acelerado ritmo con que se modifican los hábitats naturales y se pierden especies a nivel local y regional (Navarro \& Sánchez-González, 2002), hace urgente la necesidad por comprender tanto los patrones como los factores que determinan la distribución de las especies, a fin de diseñar las estrategias convenientes para su conservación (Escalante, Navarro, \& Peterson, 1998). Esta necesidad es mayor en regiones con alto número de endemismos, donde su pérdida se traduciría en extinciones globales (Forey, Humphries, \& Vane-Wright, 1994).

Nayarit, un estado del occidente de México cuya superficie representa el $1.4 \%$ del territorio nacional, es considerado una zona importante por su avifauna endémica (NavarroSigüenza et al., 2014). No obstante, son pocos los estudios realizados para comprender los patrones de distribución de la avifauna nayarita (García-Trejo \& Navarro, 2004), mismos que en su mayoría se han enfocado en las zonas costeras e insulares (i.e. Cupul-Magaña, 1999; Rebón-Gallardo, 2000). Por la limitada información sobre áreas serranas y sus aves, el objetivo de este trabajo fue describir la variación de la riqueza de especies y los ensamblajes de este grupo a lo largo de un gradiente altitudinal, con el fin de generar información que sirva como base para el diseño de estrategias para su conservación, además de conocer el patrón altitudinal de riqueza en esta zona, con el fin de contribuir a la discusión general sobre este tema a nivel global.

\section{MATERIALES Y MÉTODOS}

Área de estudio: Corresponde a la región sur del Estado de Nayarit (municipios de Bahía de Banderas, Compostela, San Pedro Lagunillas, Jala y La Yesca), caracterizada por una topografía muy accidentada, con elevaciones que van desde el nivel del mar hasta los 2760 msnm (Fig. 1; Cuadro 1). En las partes bajas predominan los climas cálido y semicálido, con temperaturas medias anuales de hasta $26{ }^{\circ} \mathrm{C} \mathrm{y}$ una precipitación media anual de 800 a 1500 mm. A partir de los $2000 \mathrm{msnm}$, el clima cambia a templado, con una temperatura media anual entre 12 y $18{ }^{\circ} \mathrm{C}$ y una precipitación anual de 800 a 1000 mm (INEGI, 2000).

Con respecto a la vegetación, las partes bajas del gradiente están cubiertas principalmente por selvas tropicales (selva mediana subcaducifolia y selva mediana subperennifolia), donde las especies arbóreas predominantes son Brosimum alicastrum, Bursera simaruba y Orbignya guacuyule (Bravo, Gómez, \& Márquez, 2013). Al aumentar la elevación, entre 


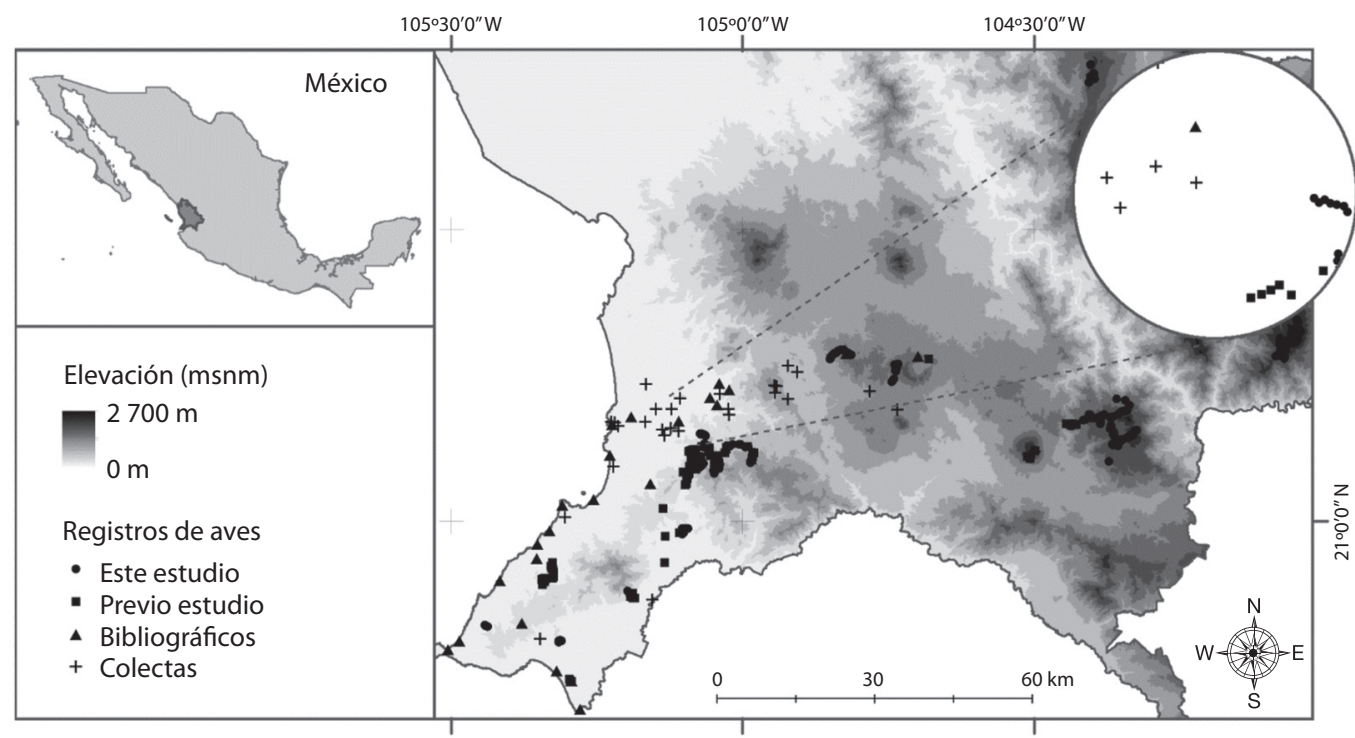

Fig. 1. Gradiente altitudinal muestreado en el Sur de Nayarit, México. Los símbolos representan los puntos de conteo realizados durante este estudio, e información obtenida de artículos científicos, catálogos de museos, y muestreos previos al área de estudio.

Fig. 1. Altitudinal gradient sampled in southern Nayarit, Mexico. Symbols represent the counting points carried out in this study, and obtained information from journal articles, museum catalogues, and previous sampling in study area.

\section{CUADRO 1}

Intervalos altitudinales y tipos de vegetación muestreados en el sur de Nayarit, México

TABLE 1

Altitudinal intervals and vegetation types sampled in Southern Nayarit, Mexico

\begin{tabular}{|c|c|c|c|}
\hline $\begin{array}{l}\text { Intervalo altitudinal } \\
\qquad(\mathrm{msnm})\end{array}$ & Municipios muestreados & Vegetación & Fechas de muestreo \\
\hline $0-300$ & Bahía de Banderas, Compostela & SMSC (32) & Dic. 2013; Ene. 2014; Sep. 2014 \\
\hline $301-600$ & Bahía de Banderas, Compostela & SMSC (14), SMSP (18) & Dic. 2013, Ene.-Mar. 2014; Ago.-Sep. 2014 \\
\hline $601-900$ & Bahía de Banderas, Compostela & SMSC (4), SMSP (16), BMM (12) & Dic. 2013; Ene. 2014; Jul.-Ago.-Sep. 2014 \\
\hline $901-1200$ & Compostela & $\operatorname{SMSP}(6), \operatorname{BMM}(26)$ & Ene.-Feb. 2014; Jul. 2014 \\
\hline $1201-1500$ & Compostela, S. Pedro Lagunillas & BMM (2), BE (30) & Ene.-Feb.-Mar. 2014; Jul.-Ago. 2014 \\
\hline $1501-1800$ & Compostela, S. Pedro Lagunillas & $\mathrm{BE}(32)$ & Mar. 2014, Jul. 2014 \\
\hline $1801-2100$ & Jala & BPE (32) & Mar. 2014, Jul. 2014 \\
\hline $2101-2400$ & La Yesca & BPE (32) & Sep. 2014, Ene. 2015 \\
\hline $2401-2700$ & La Yesca & BPE (32) & Sep. 2014, Ene. 2015 \\
\hline
\end{tabular}

$\mathrm{SMSC}=$ Selva mediana subcaducifolia; $\mathrm{SMSP}=$ Selva mediana subperennifolia; $\mathrm{BMM}=$ Bosque mesófilo de montaña; $\mathrm{BE}$ $=$ Bosque de encino; $\mathrm{BPE}=$ Bosque de pino-encino.

los 900 y 1200 msnm aparece el bosque mesófilo de montaña, distribuido principalmente en cañadas profundas y húmedas y caracterizado por especies arbóreas como Magnolia pacifica, Cornus disciflora, Inga hintonii y Quercus castanea (Babb et al., 2010). El bosque mesófilo de montaña se asocia con las selvas en su límite inferior y con el bosque de encino en su límite superior. El bosque de encino se mantiene como una comunidad distintiva hasta los $1800 \mathrm{msnm}$, donde forma asociaciones con el bosque de pino, las cuales predominan hacia las partes 
más altas del gradiente (2 $700 \mathrm{msnm})$, donde es común encontrar especies como Pinus devoniana y Pinus oocarpa (Blanco, 1994).

Toma de datos: En el periodo de diciembre 2013 a enero 2015, se realizó el muestreo de las aves a través de un gradiente altitudinal de 0 a $2700 \mathrm{msnm}$, que por cuestiones prácticas fue dividido en intervalos altitudinales de $300 \mathrm{~m}$ (Cuadro 1), una escala adecuada para identificar patrones altitudinales de riqueza (Grytnes, Beaman, Romdal, \& Rahbek, 2008). Cada intervalo altitudinal fue visitado en dos ocasiones a lo largo del ciclo anual, para tener representación de las comunidades residentes durante su periodo reproductivo y durante su periodo no reproductivo con las visitantes invernales del norte. En cada intervalo se realizaron 32 puntos de conteo de radio ilimitado que se ubicaron a $200 \mathrm{~m}$ uno de otro (Gutzwiller, 1991), con duración de 10 minutos (Hutto, Pletschet, \& Hendricks, 1986; Lynch, 1995), en los que se registraron las especies de aves de forma auditiva y visual (con la ayuda de binoculares $8 \times 42$ y mediante guías de campo (Dunn \& Alderfer, 2006; Howell \& Webb, 1995). No se contemplaron las aves acuáticas y las aves en vuelo sólo se consideraron cuando hacían uso efectivo del hábitat (i.e. golondrinas o vencejos cazando insectos). Debido a que la detección de las aves puede estar bajo la influencia de factores como las condiciones meteorológicas y los observadores (Verner, 1985), se buscó disminuir estos sesgos muestreando sólo en los días en que el tiempo fue favorable, involucrando en el muestreo a los mismos observadores y siempre entre las 06:00 y las 11:00 horas. Asimismo, para tratar de disminuir sesgos provocados por la diversidad y perturbación de los hábitats, el muestreo estuvo limitado a zonas con bajo grado de perturbación (vegetación primaria o secundaria arbórea) (Nogués-Bravo, Araújo, Romdal, \& Rahbek, 2008; Stotz, Fitzpatrick, Parker, \& Moskovits, 1996).

Con el fin de complementar el listado de especies se incluyeron registros de presencia de la Base de Datos del Museo de Zoología de la Unidad Académica de Agricultura de la Universidad Autónoma de Nayarit (Ver. 1, Nov 2015), la cual incluye información sobre: 1) artículos publicados (1941 a 2008), 2) catálogos de colecciones científicas nacionales y extranjeras (VertNet Project, http://vertnet.org, 1941 a 1971) y 3) muestreos previos en el área de estudio (2012). La información se depuró de tal forma que sólo se consideraron los registros georreferenciados y sólo se seleccionaron los registros ubicados en áreas que actualmente conservan la estructura de su vegetación, asumiendo que el hábitat presentaba mejores condiciones en el pasado. Se determinó el estatus de conservación de las especies de acuerdo a la Norma Oficial Mexicana 059 (SEMARNAT, 2010), el estatus de residencia de acuerdo a Howell y Webb (1995) y el estatus de endemismo de acuerdo a González-García y Gómez de Silva (2002).

Todos los registros de aves fueron asignados a un tipo de vegetación general. La estructura del hábitat se determinó usando un protocolo adaptado de Ralph, Geupel, Pyle, Martin y DeSante (1993), que consistió en determinar la altura promedio superior e inferior de cada estrato de vegetación, la cobertura total de cada estrato de acuerdo a la escala establecida por Braun-Blanquet (Mueller-Dombois \& Ellenberg, 1974) y el diámetro a la altura del pecho del árbol con el tronco más grueso (DAP mayor) y del árbol más delgado (DAP menor). El procedimiento anterior se llevó a cabo considerando un radio de $50 \mathrm{~m}$ alrededor de cada punto de conteo de aves, donde se contaron además el número de tocones (partes de troncos de árboles que quedan unidos por la raíz cuando los cortan) y troncos observados. Adicionalmente, considerando un radio de 100 $\mathrm{m}$, se documentó la distancia y estacionalidad de cuerpos de agua y la presencia de distintas perturbaciones antropogénicas.

Para determinar la representatividad del esfuerzo de muestreo, se realizaron curvas de rarefacción basadas en muestras para cada intervalo altitudinal y para el gradiente completo (Gotelli \& Colwell, 2001), considerando la información completa del ciclo anual. Con el programa EstimateS 9.1.0 (Colwell, 2013) 
se establecieron la riqueza observada y la estimada a partir del estimador no paramétrico Chao2, pues se ha demostrado que presenta un mejor desempeño comparado con funciones asintóticas extrapoladas u otros estimadores no paramétricos (Colwell \& Coddington, 1994; Walther \& Moore, 2005).

Para determinar el comportamiento de la riqueza a través del gradiente, se calculó la expectación estadística de la riqueza de especies (Sest [analytical]) en cada intervalo altitudinal utilizando el programa EstimateS 9.1.0 (Colwell, 2013). Ésta se calcula por medio de muestreos iterativos de todo el conjunto de muestras permitiendo las comparaciones estadísticas entre tratamientos (Gotelli \& Colwell, 2001), donde el traslape de sus intervalos de confianza al $84 \%$ equivale a pruebas estadísticas con un $\alpha$ de 0.05 (MacGregor-Fors \& Payton, 2013).

Con el fin de identificar las principales variables que afectan el comportamiento de la riqueza a través del gradiente, se realizó un árbol de regresión, una técnica estadística que permite explicar relaciones complejas no lineales entre una variable respuesta y un conjunto de variables explicativas que pueden ser numéricas o categóricas (De'Ath \& Fabricius, 2000). El árbol se construye por medio de divisiones binarias repetidas de los datos, definidas por reglas que se basan en las variables explicativas; en cada división los datos se separan en dos nodos mutuamente exclusivos (cada uno de ellos tan homogéneo como sea posible) y el proceso de división se aplica secuencialmente a cada nodo por separado. La homogeneidad de cada nodo se define por su impureza (suma de cuadrados con respecto al valor medio de la variable respuesta que caracteriza cada grupo), una medida que toma el valor de cero para nodos completamente homogéneos. Los árboles de regresión son herramientas estadísticas adecuadas para el análisis y la exploración de conjuntos de datos ecológicos (Ferenc, Sedláček, \& Fuchs, 2014; MacGregor-Fors, Ortega-Álvarez, Barrera-Guzmán, \& Sevillano, 2013) y una alternativa para muchas técnicas estadísticas tradicionales (De'Ath \& Fabricius,
2000). Para construir el árbol de regresión se consideró como variable respuesta la riqueza documentada en cada punto de conteo y como variables explicativas los datos obtenidos del muestreo de la vegetación, además de la variable bioclimática precipitación anual, la cual se obtuvo de las coberturas climáticas interpoladas por Cuervo-Robayo et al. (2013). El análisis se llevó a cabo utilizando la librería “tree” (Ripley, 2014) del paquete estadístico R (R Core Team, 2015).

Para determinar el efecto del gradiente en la composición de las especies se realizaron análisis de clasificación jerárquica sensu Villaseñor-Gómez (2008), aplicando la técnica "Two-Way Indicator Species Analysis" (TWINSPAN), la cual encuentra las relaciones entre las especies y las muestras a través de análisis de correspondencia. En un principio divide las muestras en dos grupos y después refina la clasificación a través de análisis de correspondencia sin tendencia (DCA por sus siglas en inglés), lo que le permite encontrar las especies indicadoras y asociadas de cada grupo. Con base en esas especies, el análisis divide progresivamente estos grupos en otros más pequeños hasta encontrar el límite. Una especie indicadora es la especie o grupo de especies presentes en todos los intervalos de cada piso altitudinal, mientras que una especie asociada está presente principalmente en un piso altitudinal pero puede estar en menor medida en otro (Villaseñor-Gómez, 2008). Para realizar el dendrograma de similitud entre pisos altitudinales se utilizó el índice de Sorensen (ligadura promedio) sobre matrices de datos de presencia-ausencia; el dendrograma se elaboró con el programa PC-ORD para Windows (McCune \& Mefford, 1999).

Posteriormente, con el fin de determinar si la variación en la composición avifaunística a través del gradiente responde a cambios en los tipos de vegetación, se realizó un análisis de escalamiento multidimensional no métrico (NMDS por sus siglas en inglés), una técnica multivariada de interdependencia que representa en un espacio geométrico de $k$ dimensiones las proximidades existentes entre un 
conjunto de objetos (Legendre \& Legendre, 2012; McCune, Grace, \& Urban, 2002). En este caso se representó la similitud entre la composición de especies de cada intervalo altitudinal y la composición de especies de cada tipo de vegetación en un espacio geométrico de dos dimensiones. Este análisis se realizó con la librería "vegan" (Oksanen et al., 2015) en el paquete estadístico R (R Core Team, 2015).

\section{RESULTADOS}

Se registró un total de 275 especies de aves para el sur de Nayarit, 223 especies se registraron en el trabajo de campo (esfuerzo de trabajo total de 288 puntos de conteo) y 52 especies más se agregaron como resultado de la adición de datos sobre literatura, catálogos de museos y de las visitas previas al área de estudio. El 74 $\%$ de las especies reportadas tienen residencia permanente en el Estado, mientras que el resto son migratorias de invierno. El $11 \%$ de las especies encontradas son consideradas como especies endémicas y el $15 \%$ tiene algún grado de amenaza a nivel nacional (Apéndice 1, disponible sólo en la versión de internet).

La mayoría de los valores del estimador Chao2 indican que los porcentajes de riqueza registrada por intervalo altitudinal se encontraron por encima del $65 \%$ (siendo la excepción el valor para el intervalo superior del gradiente, con $58 \%$ ); en general, el porcentaje de riqueza observada para la totalidad del gradiente fue del $84 \%$. La comparación de la expectación estadística mostró que la riqueza de especies entre los intervalos altitudinales varía a través del gradiente. En las partes bajas del gradiente (0-600 msnm) el número de especies se mantuvo relativamente alto (79), hasta alcanzar un máximo de riqueza (95) hacia las partes medias (601-1 $500 \mathrm{msnm}$ ); de aquí, la riqueza disminuyó conforme aumentó la elevación, hasta alcanzar sus valores mínimos (47) en las partes más altas (2 101-2 700 msnm; Fig. 2).

Se identificaron ocho variables con influencia determinante en el comportamiento de la riqueza de aves: precipitación anual, distancia a cuerpos de agua, límite superior

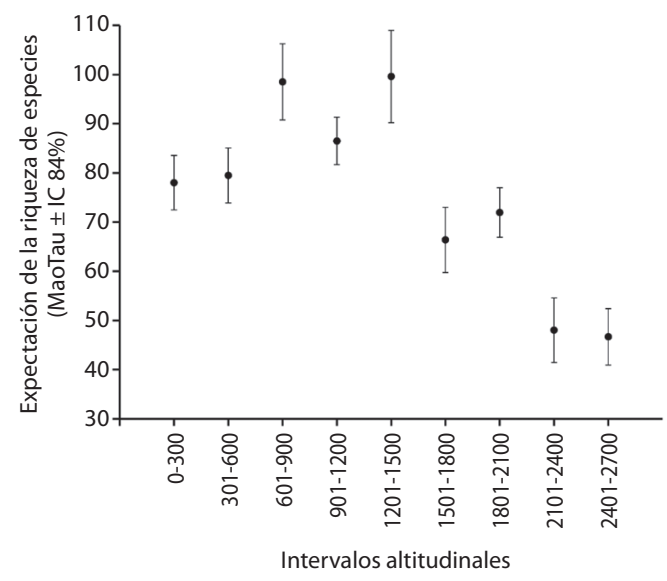

Fig. 2. Expectación estadística de la riqueza de especies de aves para nueve intervalos altitudinales del sur de Nayarit, México. Los valores fueron calculados con base en la extrapolación de 550 individuos.

Fig. 2. Expectation statistical of bird species richness to nine altitudinal intervals in southern Nayarit, Mexico. Values were calculated based on extrapolation to 550 individuals.

arbóreo, límite superior arbustivo, diámetro a la altura del pecho, cobertura del estrato arbustivo, cobertura del estrato herbáceo y número de tocones (Fig. 3). La variable con mayor influencia fue la precipitación anual; los sitios con mayor precipitación presentaron una mayor riqueza de especies y corresponden a las partes bajas-medias del gradiente (0-1 $200 \mathrm{msnm}$ principalmente). En estos sitios la riqueza máxima se alcanzó donde la distancia a cuerpos de agua es menor a $75 \mathrm{~m}$ y disminuyó en sitios que además de estar alejados de cuerpos de agua, presentaron bajas coberturas del estrato herbáceo. Por otra parte, los sitios con menores niveles de precipitación anual presentaron valores más bajos de riqueza y correspondieron a las partes medias-altas del gradiente (1 201-2 $700 \mathrm{msnm}$ principalmente). En estos sitios la riqueza de aves se concentró en lugares con un mayor límite superior arbustivo, un DAP mayor a $40.5 \mathrm{~cm}$ y un límite superior arbóreo menor a $17.5 \mathrm{~m}$, mientras que los valores más bajos de riqueza, se presentaron en lugares con un estrato arbustivo bajo, baja cobertura y la presencia de un mayor número de tocones. 


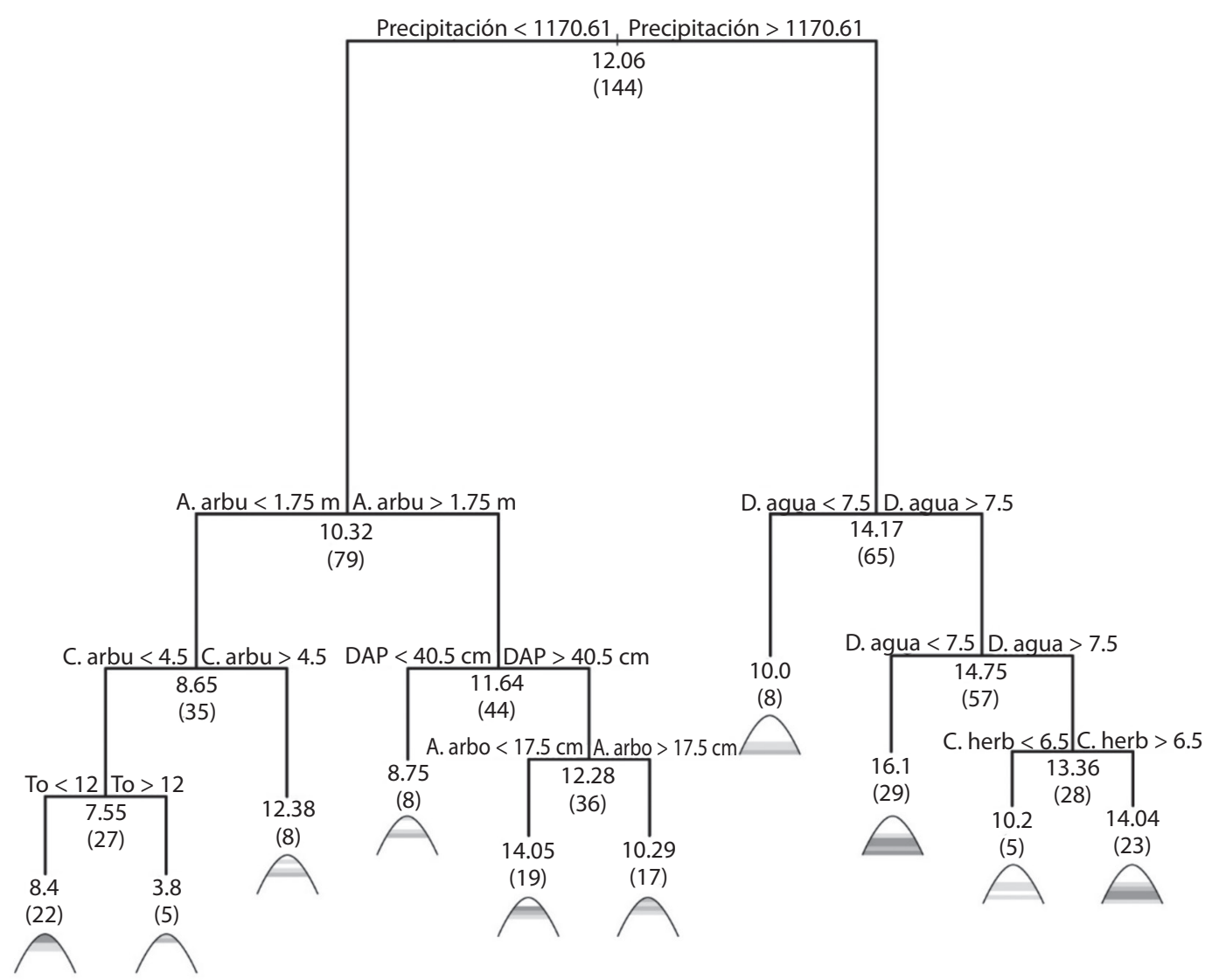

Fig. 3. Árbol de regresión de las principales variables que afectan la riqueza de aves en el sur de Nayarit, México. Precipitación $=$ precipitación anual; D. agua $=$ distancia a cuerpos de agua; A. arbo = límite superior arbóreo; A. arbu = límite superior arbustivo; DAP = diámetro a la altura del pecho; $\mathrm{C}$. arbu = cobertura del estrato arbustivo; $\mathrm{C}$. herb = cobertura del estrato herbáceo; To = número de tocones. Cada división (nodo no terminal) está señalada con el valor medio de la riqueza y el número de puntos de conteo que determinan la división (entre paréntesis); y en cada nodo terminal se muestra en el diagrama de una montaña la distribución de los puntos de conteo a través del gradiente altitudinal (la intensidad del color es proporcional al número de puntos de conteo en cada intervalo altitudinal). El árbol explica el $52.9 \%$ de la variación total de la riqueza, y la longitud vertical de cada división es proporcional a la variación explicada.

Fig. 3. Tree Regression analysis of the main variables affecting bird species richness in southern Nayarit, Mexico. Precipitación = annual precipitation; D. agua $=$ distance to water bodies; A. arbo = height of upper tree layer; A. arbu $=$ height of upper shrub layer; DAP $=$ diameter at breast height; $\mathrm{C}$. arbu = cover of shrub layer; $\mathrm{C}$. herb $=$ cover of herb layer; To $=$ number of logs. Each of the splits (nonterminal node) is labeled with the mean richness value and the number of point counts that determine the split (in parentheses), and for each of the terminal nodes the distribution of point counts on the altitudinal gradient is shown in a mountain diagram (color intensity is proportional to number of point counts in each altitudinal interval). The tree explained $52.9 \%$ of the total richness variation, and the vertical depth of each split is proportional to the variation explained.

Por otra parte, se observaron cambios en la composición avifaunística a través del gradiente, que se reflejaron en la existencia de tres pisos altitudinales (Fig. 4). El primer piso estuvo conformado por especies que se distribuyeron desde el nivel del mar hasta los $1200 \mathrm{msnm}$.
De estas especies, 13 se consideraron indicadoras de este piso (i.e. Penelope purpurascens, Campephilus guatemalensis y Eupsittula canicularis) y 18 se identificaron como especies asociadas (i.e. Chlorostilbon auriceps, Colaptes auricularis y Xiphorhynchus flavigaster; 

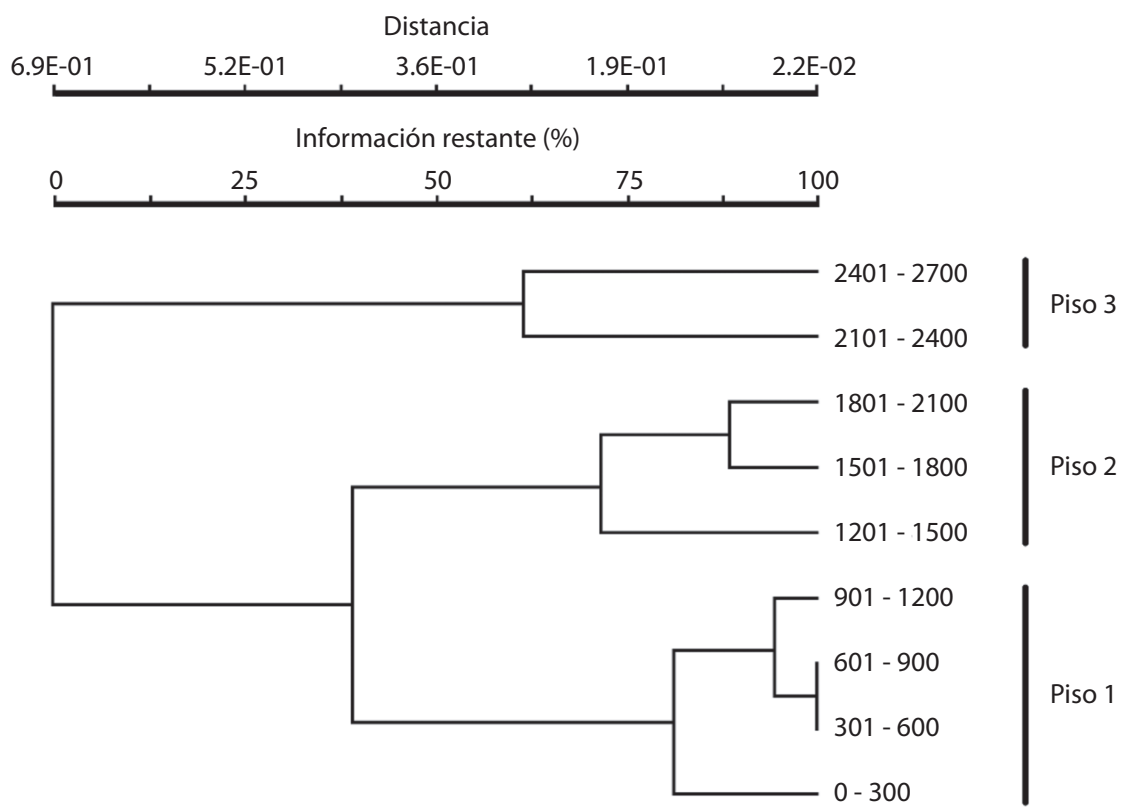

Fig. 4. Pisos altitudinales en el sur de Nayarit, México, según la composición de especies de aves de cada intervalo altitudinal. Se empleó el índice de similitud de Sorensen (ligadura promedio) y un umbral del $60 \%$ para definir los pisos altitudinales. Los números de los intervalos representan la altura en metros sobre el nivel del mar.

Fig. 4. Altitudinal associations in southern Nayarit, Mexico, according to bird species composition of each altitudinal interval. Sorensen similarity index was employed (group average) and $60 \%$ similarity threshold to define altitudinal associations. The numbers represent the meters above sea level.

Cuadro 2); el $12 \%$ de las especies de este piso fueron consideradas como endémicas y el $15 \%$ dentro de alguna categoría de amenaza.

El segundo piso lo conformaron las especies de elevaciones intermedias (1201-2 $100 \mathrm{msnm}$ ) y se caracterizó por la presencia de tres especies indicadoras: Empidonax albigularis, Vireo huttoni y Catherpes mexicanus; no presentó especies asociadas (Cuadro 2). Del total de especies registradas en este piso, el $10 \%$ fueron endémicas, mientras que otro $10 \%$ se encontraron incluidas en alguna categoría de riesgo.

El tercer piso lo conformaron únicamente especies de zonas altas (2101-2700 msnm), con nueve especies indicadoras (i.e. Cyrtonyx montezumae, Euptilotis neoxenus y Aphelocoma ultramarina), además de cuatro especies asociadas (i.e. Patagioenas fasciata, Peucedramus taeniatus e Icterus parisorum; Cuadro 2). El $12 \%$ de la especies de este piso fueron consideradas endémicas y el $15 \%$ estuvieron sujetas a alguna categoría de riesgo.

Finalmente, se observó que los cambios en la composición de las especies a través del gradiente coinciden en cierta medida con los cambios en la composición de las especies por tipo de vegetación en que se distribuyeron (Fig. 5). De esta manera se observó que la composición avifaunística del primer piso altitudinal (0-1200 msnm) fue muy similar a la agrupación formada por especies que habitan las selvas tropicales y el bosque mesófilo de montaña. Por otra parte, la composición avifaunística del segundo piso altitudinal (1201-2 $100 \mathrm{msnm}$ ) fue más parecida al grupo de especies que se distribuyen tanto en bosques de encino como en bosques de pino-encino. Finalmente, la composición avifaunística del tercer piso altitudinal $(2101-2700 \mathrm{msnm})$ tuvo una mayor similitud con las especies que habitan los bosques de pino-encino. 
CUADRO 2

Especies indicadoras y asociadas a cada piso altitudinal del sur de Nayarit, México

TABLE 2

Indicator and associated species of ech altitudinal association in southern Nayarit, Mexico

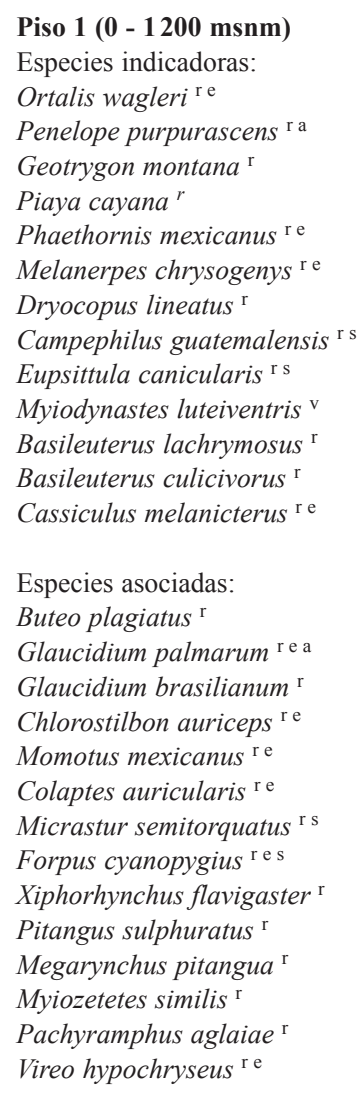

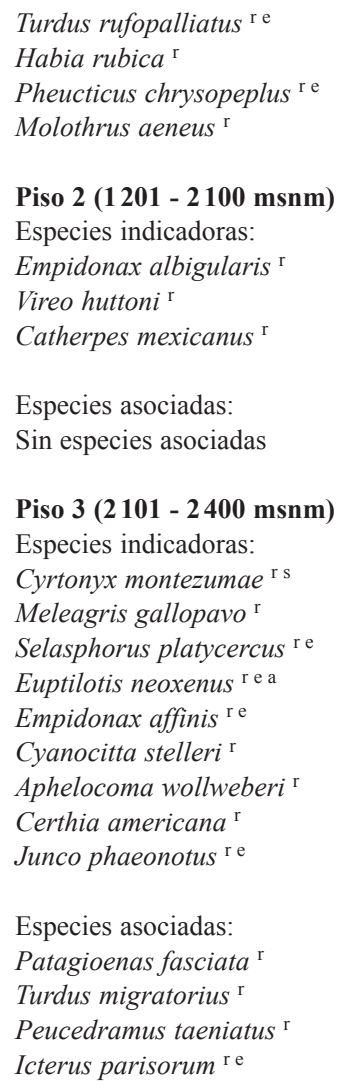

Superíndices: $\mathrm{r}=$ residente; $\mathrm{v}=$ visitante de verano; $\mathrm{e}=$ endémica; $\mathrm{s}=$ sujeta a protección especial; $\mathrm{a}=$ amenazada).

\section{DISCUSIÓN}

El conocimiento de la variación de la riqueza y la composición de las comunidades a través de gradientes altitudinales permite identificar algunos de los factores que afectan su distribución. Esta información permite comprender cómo podrían cambiar los ensamblajes de aves frente a diversos escenarios, cuáles especies y áreas serían las más afectadas, y por lo tanto a dónde deberían enfocarse los esfuerzos de conservación (Grytnes, 2003; McCain \& Grytnes, 2010; Rahbek, 1995).
En el gradiente estudiado al sur de Nayarit, el patrón encontrado fue el de "mayor riqueza en bajas altitudes con incremento en elevaciones intermedias", patrón similar al que ha sido reportado en aves por varios estudios previos (i.e. Madagascar, Hawkins, 1999; oriente de los Andes en Bolivia, Herzog et al., 2005; Andes colombianos, Kattan \& Franco, 2004), en contraste con el patrón encontrado por Navarro (1992) para un gradiente en el estado de Guerrero, quien identificó un patrón de disminución lineal de la riqueza. 


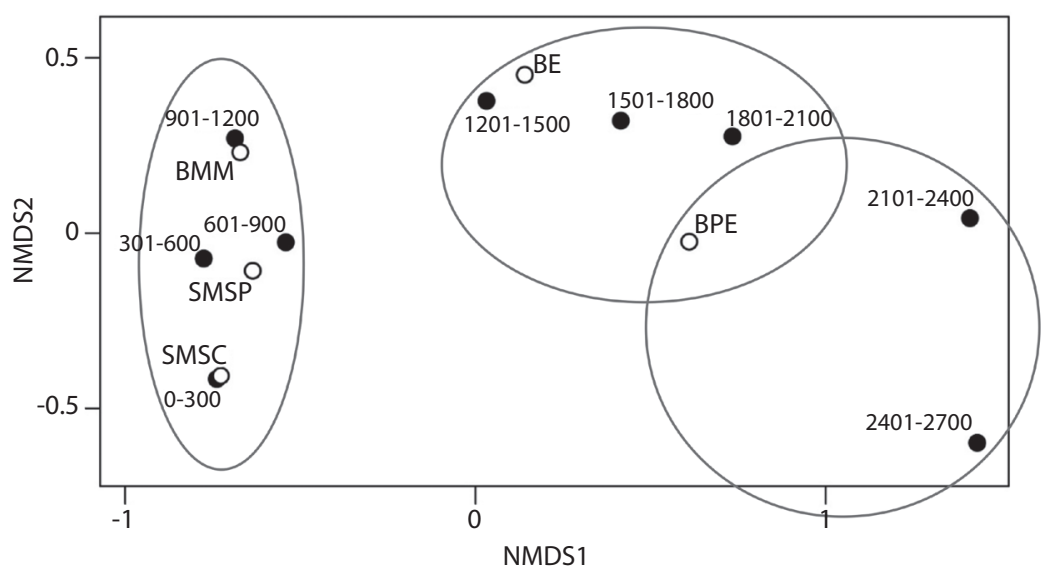

Fig. 5. Escalamiento multidimensional no métrico que muestra la relación entre la composición de los ensamblajes de aves en los intervalos altitudinales y los tipos de vegetación del gradiente en el sur de Nayarit, México. Medida de distancia $=$ Jaccard. Desajuste $<0.05$. Círculos rellenos representan intervalos altitudinales y círculos vacíos representan tipos de vegetación. $\mathrm{SMSC}=$ selva mediana subcaducifolia; $\mathrm{SMSP}=$ selva mediana subperennifolia; $\mathrm{BMM}=$ bosque mesófilo de montaña; $\mathrm{BE}=$ bosque de encino; BPE = bosque de pino-encino. Es notable la cercanía de los puntos que representan los intervalos y los tipos de vegetación a los que corresponden.

Fig. 5. Graph of Nonmetric Multidimensional Scaling showing the relationship between the composition of bird communities at the different elevation intervals and vegetation types of the altitudinal gradient in southern Nayarit, Mexico. Distance measure $=$ Jaccard. Stress $<0.05$. Filled circles represent altitudinal intervals, and unfilled circles represent vegetation types. $\mathrm{SMSC}=$ deciduous forest; $\mathrm{SMSP}=$ evergreen forest; $\mathrm{BMM}=$ cloud forest; $\mathrm{BE}=$ oak forest; $\mathrm{BPE}=$ pine-oak forest. Note the closeness of the points representing intervals and the corresponding vegetation types.

En regiones tropicales, diversos estudios han encontrado que la riqueza de especies está limitada principalmente por restricciones climáticas asociadas con la precipitación, que tiende a concentrarse en las partes bajas y medias de los gradientes altitudinales (Barry, 2008; McCain \& Grytnes, 2010) permitiendo el establecimiento de zonas de mayor productividad, y por lo tanto de mayor riqueza de especies (Nor, 2001). En este estudio, las áreas de mayor riqueza en el gradiente, representadas por las zonas donde existe el bosque mesófilo, presentan los valores más altos de precipitación y una mayor disponibilidad de cuerpos de agua, por lo que es probable que la riqueza esté respondiendo positivamente a zonas de mayor productividad. No obstante, uno de los sitios de mayor riqueza de especies (con vegetación de encinar, 1201-1500 msnm) presenta niveles de precipitación más bajos, por lo que otros factores podrían tener influencia en el comportamiento de la riqueza a través del gradiente.
En estos mismos intervalos se encuentran las zonas de transición entre el bosque mesófilo y bosque de encino, donde especies de tierras bajas y altas alcanzan su límite superior e inferior, respectivamente. Este comportamiento ha sido previamente documentado en aves por Medina-Macías y colaboradores (MedinaMacías, González-Bernal, \& Navarro-Sigüenza, 2010), quienes argumentan que el bosque de encino, debido a su estructura y ubicación en los gradientes, pudiera funcionar como una barrera que ha limitado el movimiento de especies entre tierras bajas y altas, y por lo tanto ha actuado como un área de recambio para especies congenéricas, efecto que puede ser evidente también en este estudio (i.e. Glaucidium brasilianum vs. G. gnoma, Melanerpes chrysogenys vs. M. formicivorus, Turdus rufopalliatus vs. T. migratorius).

Una explicación alternativa está dada por las características estructurales del hábitat (MacArthur, 1964; MacArthur \& MacArthur, 
1961), pues se ha documentado que aspectos de la vegetación como estratos altos, amplia cobertura y troncos más gruesos, pueden conferir a los hábitats una estructura más compleja que propicia mayor abundancia de recursos alimenticios y una mayor riqueza de especies (Ferenc et al., 2014; MacGregor-Fors et al., 2013; Mörtberg, 2001; Murgui, 2007). En este estudio, la riqueza de especies responde positivamente a estos aspectos de la estructura del hábitat, sin embargo, el intervalo 1201-1500 presenta características que por el contrario sugieren que se trata de un sitio perturbado (bajos estratos arbóreos y troncos delgados), por lo que su alta riqueza no puede ser explicada por las mismas características. En este sentido, se ha documentado que la perturbación puede generar un mosaico de hábitats o estados sucesionales que pueden incrementar la riqueza de especies (Angelstam, 1998), por lo que es posible que la perturbación esté jugando un papel determinante.

Otros estudios han sugerido que la riqueza máxima en las elevaciones medias de los gradientes altitudinales está determinada por una sobreposición de los rangos de distribución de las especies en esas elevaciones ("efecto del dominio medio"), como resultado de las restricciones geométricas impuestas por las montañas (Colwell \& Hurtt, 1994). Esta hipótesis predice que un modelo nulo de distribución de especies a través de un gradiente altitudinal producirá una curva simétrica en forma de joroba (Colwell, Rahbek, \& Gotelli, 2004); no obstante, no explica completamente las curvas asimétricas como la reportada en este estudio, donde otros factores biológicos y ambientales tienen un mayor efecto sobre el patrón de riqueza (Colwell \& Hurtt, 1994).

Por otra parte, nuestros análisis muestran que las aves responden a cambios a través del gradiente y restringen su distribución en tres diferentes pisos altitudinales, que coinciden en gran medida con los grupos de vegetación. Por lo tanto, se sugiere que la vegetación es uno de los factores que moldea la composición de los ensamblajes de aves, ya sea mediante cambios en su estructura y fenología (Robinson
\& Holmes, 1984), composición (Lee \& Rotenberry, 2005), o variación en la disponibilidad de recursos (Blake \& Loiselle, 2000; Martínez $\&$ Rechberger, 2007).

De esta manera, el primer piso altitudinal se constituye por aves cuyo hábitat se limita a bosques tropicales y bosques mesófilos de montaña, los cuales presentan una estructura y composición muy similar que los distingue de los otros tipos de vegetación. Estos bosques muestran un sotobosque denso cuando están conservados (o se componen por arbustos de café de sombra cuando son intervenidos por el hombre) y un estrato arbóreo diverso (Challenger, 2003). Algunas de las aves se restringen a este piso, mientras que otras especies pueden ampliar su distribución al siguiente, aunque en menor medida. Por ejemplo, E. canicularis se considera indicadora debido a que habita principalmente selvas tropicales y alcanza su límite altitudinal en el bosque mesófilo de montaña a 1100 msnm. De acuerdo con Collar (1997), su dieta consiste en frutos y semillas de especies arbóreas restringidas al primer piso altitudinal, como Ficus, Bursera, Brosimum, Inga y Ceiba, por lo que es probable que su distribución esté limitada por la composición de la vegetación de este piso altitudinal y los límites de distribución de las especies de las que se alimenta.

El segundo piso altitudinal está conformado por aves que habitan principalmente los bosques de encino y de pino-encino. La escasez de especies indicadoras sugiere que este piso es un área de transición cuya composición avifaunística está conformada por una mezcla de especies tanto de bajas como altas elevaciones. Sus especies indicadoras E. albigularis, $V$. huttoni y C. mexicanus, sugieren la dominancia de hábitats arbóreos abiertos con crecimiento secundario arbustivo (Brewer \& Orenstein, 2010; Fitzpatrick et al., 2004; Kroodsma \& Brewer, 2005), características que describen la estructura de la vegetación de este piso y lo distinguen del tercer piso altitudinal.

En el tercer piso altitudinal, el cambio en la composición de la avifauna coincide con un cambio en la vegetación a exclusivamente bosques de pino-encino. A diferencia del piso 
anterior, se trata de bosques que muestran aparentemente bajo grado de perturbación y mayor complejidad estructural, lo cual se confirma por la presencia de aves como E. neoxenus y Certhia americana, especies que se limitan $\mathrm{a}$ áreas extensas de bosques maduros, densos y con abundancia de árboles muertos en pie (Collar, 2001; Harrap, 2008).

Los resultados de este estudio muestran que la riqueza y composición de especies de aves cambian a través del gradiente altitudinal, influenciadas por variables como la precipitación, la vegetación y su estructura. Es probable que la respuesta positiva de la riqueza se de en términos de las áreas de mayor productividad, además de tener una influencia importante por parte de la perturbación ecológica; a pesar de que este estudio se diseñó tratando de evitar muestrear sitios con alto grado de perturbación, existen evidencias que pueden mostrar sus efectos. Considerando lo anterior, la protección de las comunidades vegetales debería ser un eje fundamental en las estrategias de conservación y deberán tomar en cuenta, tanto el endemismo como la riqueza de especies amenazadas que se mantienen a través del gradiente, señalando la importancia de considerar la totalidad del gradiente altitudinal para la conservación de estas especies. Las instancias tomadoras de decisiones deberán considerar estas relaciones con el fin de diseñar las estrategias adecuadas para la conservación de las aves de Nayarit.

La respuesta de la riqueza a lo largo de gradientes es el resultado de la interacción de múltiples variables y procesos que no han sido considerados. Es complejo definir enfoques en los que la imposibilidad de control de las variables estimadas deja muchas preguntas sin respuesta. El estudio de otros procesos que actúan de manera sinérgica podría ayudar a entender de mejor manera el comportamiento de los patrones de riqueza y composición de las comunidades, por ejemplo el caso de dinámicas metapoblacionales, competencia y otras interacciones bióticas (Terborgh, 1977; Lomolino, 2001; McCain, 2009). Análisis adicionales que consideren la evaluación de estos procesos podrían revelar nueva información sobre los factores que influyen y moldean la distribución de la riqueza de las especies en clinas de elevación.

\section{AGRADECIMIENTOS}

Este estudio fue posible gracias al financiamiento del Proyecto PROMEP/103.5/13/6621 de Apoyo a la Incorporación de Nuevos PTC de la SEP. Nidia Jaime agradece a CONACYT por la beca otorgada (292953) y al Posgrado en Ciencias Biológico Agropecuarias. Además agradecemos a los estudiantes del Museo de Zoología de la Universidad Autónoma de Nayarit, por haber participado en el trabajo de campo y en la digitalización de la información generada. Un especial agradecimiento a las personas que nos ayudaron como guías locales en cada uno de los sitios de trabajo.

\section{RESUMEN}

Los estudios sobre gradientes altitudinales han permitido examinar cambios en la riqueza y composición de los ensamblajes de aves, lo que hace posible obtener información sobre las variables ambientales que determinan su distribución y factores que definen la estructura. En este trabajo se describe la variación en la riqueza y composición de los ensamblajes de aves a través de un gradiente altitudinal al sur de Nayarit. Para analizar el comportamiento de la riqueza en el gradiente se obtuvo información mediante puntos de conteo en nueve intervalos altitudinales (separados cada $300 \mathrm{~m}$ de altitud) desde el nivel del mar hasta los $2700 \mathrm{msnm}$. Se estandarizó el esfuerzo de muestreo y se realizaron curvas de rarefacción, y los cambios en la composición de especies se analizaron mediante análisis de clasificación jerárquica utilizando la técnica TWINSPAN. Para identificar las variables asociadas con los cambios en riqueza se analizó el efecto de la precipitación y de la estructura del hábitat mediante árboles de regresión. Para determinar si los cambios en composición responden a cambios en los tipos de vegetación se realizó un análisis de escalamiento multidimensional no métrico (NMDS). La riqueza de especies varió de forma significativa a través del gradiente: fue alta en las partes bajas del gradiente, alcanzó su valor máximo en la parte media y disminuyó monotónicamente con la elevación. Las especies respondieron a cambios en el gradiente y se agruparon en tres pisos altitudinales. Los análisis sugieren que los cambios en la riqueza y composición de las especies están influenciados por la vegetación, su estructura y precipitación, así como por diversos aspectos relacionados con características del hábitat y la perturbación. Estos aspectos deberán ser tomados 
en cuenta con el fin de diseñar estrategias adecuadas para la conservación de las aves de Nayarit.

Palabras clave: patrones de distribución, gradientes altitudinales, comunidades de aves, riqueza de especies, endemismo.

\section{REFERENCIAS}

Angelstam, P. K. (1998). Maintaining and restoring biodiversity in European boreal forests by developing natural disturbance regimes. Journal of Vegetation Science, 9, 593-602.

Babb, K. A., Blanco, J. M., González, R. E., Navarrete, J. A., Téllez, O., \& Vega, R. (2010). Serranías de Nayarit. In CONABIO (Ed.), El bosque mesófilo de montaña en México: amenazas y oportunidades para su conservación y manejo sostenible (pp. 50-59). México, D.F.: Comisión Nacional para el Conocimiento y Uso de la Biodiversidad.

Barry, R. G. (2008). Mountain weather and climate. New York: Cambridge University Press.

Blake, J. G., \& Loiselle, B. A. (2000). Diversity of birds along an elevational gradient in the Cordillera Central, Costa Rica. The Auk, 117, 663-686.

Blanco, J. M. (1994). Vegetación de la Sierra de San Juan, Nayarit, México (Tesis de Licenciatura). Universidad Nacional Autónoma de México, México.

Bravo, O., Gómez, C., \& Márquez, A. R. (2013). Composición florística del Ejido de Sayulita, Bahía de Banderas, Nayarit, México: Un análisis espacial. Revista Bio Ciencias, 2, 172-188.

Brewer, D., \& Orenstein, R. (2010). Family Vireonidae (Vireos). In J. del Hoyo, A. Elliott, \& D. Christie (Eds.), Handbook of the birds of the world (pp. 378439). Barcelona: Lynx Edicions.

Cadena, C. D., Kozak, K. H., Gómez, J. P., Parra, J. L., McCain, C. M., Bowie, R. C. K., ... Graham, C. H. (2012). Latitude, elevational climatic zonation and speciation in New World vertebrates. Proceedings of the Royal Society, 279, 194-201.

Colwell, R. K. (2013). EstimateS: statistical estimation of species richness and shared species from samples (Versión 9.1.0). Recuperado de http://viceroy.eeb. uconn.edu/estimates/

Colwell, R. K., \& Coddington, J. A. (1994). Estimating terrestrial biodiversity through extrapolation. Philosophical Transactions of the Royal Society B, 345, 101-118.

Colwell, R. K., \& Hurtt, G. C. (1994). Nonbiological gradients in species richness and a spurious Rapoport effect. The American Naturalist, 144, 570-595.
Colwell, R. K., Rahbek, C., \& Gotelli, N. J. (2004). The mid-domain effect and species richness patterns: what have we learned so far? The American Naturalist, 163, 1-23.

Collar, N. J. (1997). Family Psittacidae (Parrots). In J. del Hoyo, A. Elliott, \& J. Sargatal (Eds.), Handbook of the birds of the world (pp. 280-477). Barcelona: Lynx Edicions.

Collar, N. J. (2001). Family Trogonidae (Trogons). In J. del Hoyo, A. Elliott, \& J. Sargatal (Eds.), Handbook of the birds of the world (pp. 80-129). Barcelona: Lynx Edicions.

Cuervo-Robayo, A. P., Téllez-Valdés, O., Gómez-Albores, M. A., Venegas-Barrera, C. S., Manjarrez, J., \& Martínez-Meyer, E. (2013). An update of high-resolution monthly climate surfaces for Mexico. International Journal of Climatology, 34, 2427-2437.

Cupul-Magaña, F. G. (1999). La laguna El Quelele, Nayarit, México, como hábitat de aves acuáticas. Ciencia y Mar, 3, 25-32.

Challenger, A. (2003). Conceptos generales acerca de los ecosistemas templados de montaña de México y su estado de conservación. En Ó. Sánchez, E. Vega, E. Peters, \& O. Monróy-Vilchis (Eds.), Conservación de ecosistemas templados de montaña en México (pp. 17-44). México: INE, SEMARNAT.

De'Ath, G., \& Fabricius, K. (2000). Classification and regression trees: a powerful yet simple technique for ecological data analysis. Ecology, 81, 3178-3192.

Dunn, J., \& Alderfer, J. (2006). Field guide to the birds of North America. Washington, D.C.: National Geographic.

Escalante, P., Navarro, A. G., \& Peterson, A. T. (1998). Un análisis geográfico, ecológico e histórico de la diversidad de aves terrestres de México. En T. Ramamoorthy, R. Bye, A. Lot, \& J. Fa (Eds.), Diversidad biológica de México: orígenes y distribución (pp. 279-304). México, D.F.: Instituto de Biología, Universidad Nacional Autónoma de México.

Ferenc, M., Sedláček, O., \& Fuchs, R. (2014). How to improve urban greenspace for woodland birds: site and local-scale determinants of bird species richness. Urban Ecosystems, 17, 625-640.

Fitzpatrick, J., Bates, J., Bostwick, K., Caballero, I., Clock, B., Farnsworth, A., . . . Zimmer, K. (2004). Family Tyrannidae (Tyrant-flycatchers). In J. del Hoyo, A. Elliott, \& D. Christie (Eds.), Handbook of the birds of the world (pp. 170-462). Barcelona: Lynx Edicions.

Forey, P. L., Humphries, C., \& Vane-Wright, R. (1994). Systematics and conservation evaluation. Oxford, New York: Oxford University Press. 
García-Trejo, E., \& Navarro, A. (2004). Patrones biogeográficos de la riqueza de especies y el endemismo de la avifauna en el oeste de México. Acta Zoológica Mexicana, 20, 167-185.

Gaston, K. J. (2000). Global patterns in biodiversity. Nature, 405, 220-227.

González-García, F., \& Gómez de Silva, H. (2002). Especies endémicas: riqueza, patrones de distribución y retos para su conservación. En H. Gómez de Silva \& A. Oliveras de Ita (Eds.), Conservación de aves: experiencias en México (pp. 150-194). México, D.F. Sociedad para el Estudio y Conservación de las Aves en México AC.

Gotelli, N. J., \& Colwell, R. K. (2001). Quantifying biodiversity: procedures and pitfalls in the measurement and comparison of species richness. Ecology Letters, $4,379-391$

Grytnes, J. A. (2003). Species-richness patterns of vascular plants along seven altitudinal transects in Norway. Ecography, 26, 291-300.

Grytnes, J. A., Beaman, J. H., Romdal, T. S., \& Rahbek, C. (2008). The mid-domain effect matters: simulation analyses of range-size distribution data from Mount Kinabalu, Borneo. Journal of Biogeography, $35,2138-2147$.

Gutzwiller, K. (1991). Estimating winter species richness with unlimited-distance point counts. The Auk $108,853-862$.

Harrap, S. (2008). Family Certhiidae (Treecreepers). In J. del Hoyo, A. Elliott, \& D. Christie (Eds.), Handbook of the birds of the world (pp. 166-179). Barcelona: Lynx Edicions.

Hart, P. J., Woodworth, B. L., Camp, R. J., Turner, K., McClure, K., Goodall, K., . . . Samuel, M. (2011). Temporal variation in bird and resourse abundance across an elevational gradient in Hawaii. The Auk, $128,113-126$.

Hawkins, A. F. (1999). Altitudinal and latitudinal distribution of east Malagasy forest bird communities. Journal of Biogeography, 26, 447-458.

Herzog, S. K., Kessler, M., \& Bach, K. (2005). The elevational gradient in Andean bird species richness at the local scale: a foothill peak and a high-elevation plateau. Ecography, 28, 209-222.

Howell, S., \& Webb, S. (1995). A guide to the birds of Mexico and Northern Central America. Oxford, New York: Oxford University Press.

Hutto, R., Pletschet, S., \& Hendricks, P. (1986). A fixedradius point count method for nonbreeding and breeding season use. The Auk, 103, 593-602.
INEGI. (2000). Sintesis de información geográfica del estado de Nayarit. México, D.F.: Instituto Nacional de Estadística, Geografía e Informática.

Kattan, G. H., \& Franco, P. (2004). Bird diversity along elevational gradients in the Andes of Colombia: area and mass effects. Global Ecology and Biogeography, $13,451-458$.

Koleff, P., Soberón, J., Arita, H. T., Dávila, P., FloresVillela, O., Golubov, J., ... \& Rodríguez, P. (2008). Patrones de diversidad espacial en grupos selectos de especies. En J. Soberón, G. Halffter, \& J. LlorenteBousquets (Eds.), Capital Natural de México. Conocimiento actual de la biodiversidad (pp. 323-364). Distrito Federal, México: Comisión Nacional para el Conocimiento y Uso de la Biodiversidad.

Kroodsma, D., \& Brewer, D. (2005). Family Troglodytidae (Wrens). In J. del Hoyo, A. Elliott, \& D. Christie (Eds.), Handbook of the birds of the world (pp. 356447). Barcelona: Lynx Edicions.

Lee, P. Y., \& Rotenberry, J. T. (2005). Relationships between bird species and tree species assemblages in forested habitats of eastern North America. Journal of Biogeography, 32, 1139-1150.

Legendre, P., \& Legendre, L. (2012). Numerical ecology. Oxford, New York: Elsevier.

Lomolino, M. V. (2001). Elevation gradients of speciesdensity: historical and prospective views. Global Ecology and Biogeography, 10, 3-13.

Lynch, J. F. (1995). Effects of point count duration, timeof-day, and aural stimuli on detectability of migratory and resident bird species in Quintana Roo, Mexico. USDA Forest Service, General Technical Reports, 149, 1-6.

MacArthur, R. H. (1964). Environmental factors affecting bird species diversity. The American Naturalist, 98, 387-397.

MacArthur, R. H., \& MacArthur, J. W. (1961). On bird species diversity. Ecology, 42, 594-598.

MacGregor-Fors, I., Ortega-Álvarez, R., Barrera-Guzmán, A., Sevillano, L. \& del-Val, E. (2013). Tama-risk? Avian responses to the invasion of saltcedars (Tamarix ramosissima) in Sonora, Mexico. Revista Mexicana de Biodiversidad, 84, 1284-1291.

MacGregor-Fors, I., \& Payton, M. E. (2013). Contrasting diversity values: statistical inferences based on overlapping confidence intervals. PLOS ONE, 8, 1-4.

Martínez, O., \& Rechberger, J. (2007). Características de la avifauna en un gradiente altitudinal de un bosque nublado andino en La Paz, Bolivia. Revista Peruana de Biología, 14, 225-236. 
McCain, C. M. (2009). Global analysis of bird elevational diversity. Global Ecology and Biogeography, $18,346-360$.

McCain, C. M., \& Grytnes, J. A. (2010). Elevational gradients in species richness Encyclopedia of Life Sciences. Chichester: John Wiley \& Sons, Ltd.

McCune, B., Grace, J. B., \& Urban, D. L. (2002). Analysis of ecological communities. Gleneden Beach, Oregon: MjM software design.

McCune, B., \& Mefford, M. (1999). PC-ORD. Multivariate Analysis of Ecological Data (Versión 4.10). Gleneden Beach, Oregon, USA: MjM Software Design.

Medina-Macías, M. N., González-Bernal, M., \& NavarroSigüenza, A. (2010). Distribución altitudinal de las aves en una zona prioritaria en Sinaloa y Durango, México. Revista Mexicana de Biodiversidad, $81,487-503$.

Mörtberg, U. (2001). Resident bird species in urban forest remnants; landscape and habitat perspectives. Landscape Ecology, 16, 193-203.

Mueller-Dombois, D., \& Ellenberg, H. (1974). Aims and methods of vegetation ecology. New York: John Wiley \& Sons, Ltd.

Murgui, E. (2007). Factors influencing the bird community of urban wooded streets along an annual cycle. Ornis Fennica, 84, 66-77.

Navarro, A. G. (1992). Altitudinal distribution of birds in the Sierra Madre del Sur, Guerrero, México. The Condor, 94, 29-39.

Navarro-Sigüenza, A. G., Gallardo, M. F. R., Martínez, A. G., Peterson, T., García, H. B., \& González, L. S. (2014). Biodiversidad de aves en México. Revista Mexicana de Biodiversidad, 85, 476-495.

Navarro, A. G., \& Sánchez-González, L. A. (2002). La diversidad de las aves. En H. Gómez de Silva, \& A. Oliveras de Ita (Eds.), Conservación de aves: experiencias en México (pp. 24-85). México, D. F: Sociedad para el Estudio y Conservación de las Aves en México AC.

Nogués-Bravo, D., Araújo, M. B., Romdal, T., \& Rahbek, C. (2008). Scale effects and human impact on the elevational species richness gradients. Nature, 453, 216-219.

Nor, S. M. D. (2001). Elevational diversity patterns of small mammals on Mount Kinabalu, Sabah, Malaysia. Global Ecology and Biogeography, 10, 41-62.

Oksanen, J., Guillaume, F., Kindt, R., Legendre, P., Minchin, P., O’Hara, B., ... Wagner, H. (2015). Vegan: community ecology package (Versión 2.2.1). Recuperado de https://cran.r-project.org/web/packages/vegan/
R Core Team. (2015). R: A language and environment for statistical computing. Vienna, Austria: R Foundation for Statistical Computing. Recuperado de http:// www.R-project.org/

Rahbek, C. (1995). The elevational gradient of species richness: a uniform pattern? Ecography, 18, 200-205.

Ralph, C., Geupel, G., Pyle, P., Martin, T., \& DeSante, D. (1993). Handbook of field methods for monitoring landbirds. Albany, California: Pacific Southwest Research Station Albany.

Rebón-Gallardo, F. (2000). Distribución, abundancia y conservación de la avifauna de las islas Marietas, Nayarit, México. Anales del Instituto de Biología UNAM Serie Zoología, 71, 59-88.

Ripley, B. (2014). Tree: classification and regression trees (Versión 1.0.35). Recuperado de http://CRAN.Rproject.org/package $=$ tree.

Robinson, S. K., \& Holmes, R. T. (1984). Effects of plant species and foliage structure on the foraging behavior of forest birds. The Auk, 101, 672-684.

SEMARNAT. (2010). Norma Oficial Mexicana NOM059-SEMARNAT-2010, Protección ambiental-Especies nativas de México de flora y fauna silvestres-Categorías de riesgo y especificaciones para su inclusión, exclusión o cambio-Lista de especies en riesgo. México, D.F.: Diario Oficial de la Federación.

Stotz, D. F., Fitzpatrick, J. W., Parker, T. A., \& Moskovits, D. K. (1996). Neotropical birds: ecology and conservation. Chicago: University of Chicago Press.

Terborgh, J. (1977). Bird species diversity on an andean elevational gradient. Ecology, 58, 1007-1019.

Verner, J. (1985). Assessment of counting techniques. In R. F. Johnston (Ed.), Current ornithology (pp. 247-302). USA: Springer US.

Villaseñor-Gómez, J. F. (2008). Habitat use of wintering bird communities in Sonora, México: the importance of riparian habitats. Studies in Avian Biology, $37,53-68$.

Walther, B. A., \& Moore, J. L. (2005). The concepts of bias, precision and accuracy, and their use in testing the performance of species richness estimators, with a literature review of estimator performance. Ecography, 28, 815-829.

Wilson, R. E., Valqui, T. H., \& McCracken, K. G. (2010). Ecogeographic variation in cinnamon teal (Anas cyanoptera) along elevational and latitudinal gradients. Ornithological Monographs, 67, 141-161. 
